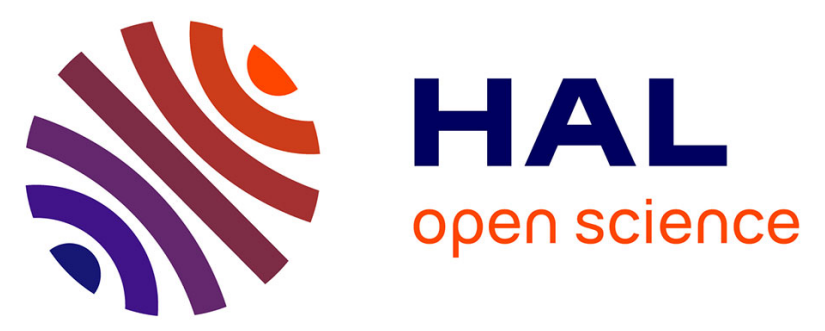

\title{
Charge-coupled devices combined with centroid algorithm for laser beam deviation measurements compared to position sensitive device
}

Bo Hou, Zong Yan Wu, Jean-Louis de Bougrenet de La Tocnaye, Philippe Grosso

\section{To cite this version:}

Bo Hou, Zong Yan Wu, Jean-Louis de Bougrenet de La Tocnaye, Philippe Grosso. Charge-coupled devices combined with centroid algorithm for laser beam deviation measurements compared to position sensitive device. Optical Engineering, 2011, 50 (3), 10.1117/1.3553479 . hal-00704225

\section{HAL Id: hal-00704225 \\ https://hal.science/hal-00704225}

Submitted on 5 Jun 2012

HAL is a multi-disciplinary open access archive for the deposit and dissemination of scientific research documents, whether they are published or not. The documents may come from teaching and research institutions in France or abroad, or from public or private research centers.
L'archive ouverte pluridisciplinaire HAL, est destinée au dépôt et à la diffusion de documents scientifiques de niveau recherche, publiés ou non, émanant des établissements d'enseignement et de recherche français ou étrangers, des laboratoires publics ou privés. 


\section{Charge-coupled devices combined with centroid algorithm for laser beam deviation measurements compared to position sensitive device}

\author{
Bo Hou \\ Z. Y. Wu \\ J. L. de Bougrenet de la Tocnaye \\ P. Grosso \\ Telecom Bretagne \\ Optics Department \\ Technopôle Iroise 29238 \\ Brest, France \\ E-mail: bo.hou@telecom-bretagne.eu
}

20

\section{${ }_{27} 1$ Introduction}

28 Since laser technology has been developed for years, it has 29 been widely used in different applications, one of which is so to measure physical parameters by detecting the laser beam 31 deviation. ${ }^{1,2}$ In this framework, a position sensitive detector 32 (PSD) is an optical sensor that can measure a light spot loca33 tion in one or two dimensions on a sensor surface. In 1957, Wallmark ${ }^{3}$ first introduced the lateral-effect photodiode PSD which has been extensively developed, including in performance analysis, ${ }^{4}$ signal processing methods,,${ }^{5,6}$ and various applications. As an analogue device, a PSD provides high sensitivity, short response time and independence from spot light size, shape and intensity. Due to these features, a PSD has been used for various laser beam deviation measurement systems. However, the PSD analogue output signal makes it hard to benefit from modern post-processing techniques. In 1969, the charge-coupled device (CCD) was invented by Boyle and Smith, recording illumination in a pixel-based image. With the development of image post-processing techniques, the CCD becomes a way to measure laser spot deviations. Compared to analogue PSD, the pixel-based CCD does not directly provide information on position, but rather on laser spot shape and intensity. The position information can then be retrieved by different post-processing algorithms. This feature makes the pixel-based image sensor extremely flexible for laser beam deviation detection, with more ca53 pacity to extract additional useful information. Our previous

0091-3286/2011/\$25.00 @ 2011 SPIE work involved developing a refractometer to measure the salinity of seawater based on measuring the laser beam deviation with a one-dimensional(1D) PSD. ${ }^{7}$ The resolution of measuring salinity reaches $0.002 \mathrm{~g} / \mathrm{kg}$ with the measurement range from 0 to $40 \mathrm{~g} / \mathrm{kg}$. Besides the salinity, other quantities of seawater, for example, the turbidity, are very important for the oceanology. This information, which is highly associated with the power distribution of laser spot, cannot be retrieved from neither 1D PSD nor two-dimensional(2D) PSD, for example, the quadrant photodiode. ${ }^{8}$ Because of this, the CCD, which is sensitive to the power distribution of light, is considered to be a replacement of the PSD. To achieve this, it is necessary to have a performance comparison between a CCD-based system and a PSD-based system, and prove that a CCD-based system could at least obtain the same resolution in laser beam deviation measurement.

To retrieve the position information from the images captured by a CCD, a localization method is needed to be applied to the images. Several previous researches involved different localization algorithms. Canabal et al. ${ }^{2}$ compared three localization algorithms: centroid, Gaussian fitting, and Fourier transform. Welch ${ }^{9}$ worked on the effects of the window size and the shape on the centroid algorithm. Bobroff's article $^{10}$ describes the theoretical limits on the ability to locate a signal position. The comparison between a quadrant diode-based system and a CMOS-based system was studied by Scott et al. ${ }^{8}$ Other researches on the CCD-based laser beam deviation measurement has focused on the comparison among several post-processing techniques ${ }^{11}$ and estimated those algorithm accuracies, ${ }^{12,13}$ including system error and 
84 noise, to improve accuracy. ${ }^{14-17}$ Because a PSD actually 85 gives the gravity center of a laser spot, in this paper, the 86 centroid algorithm, which is also calculating the gravity cen87 ter, is combined with a CCD to compare with the state-of88 the-art PSD-based system.

A 1D-PSD transfers the laser beam illumination into two photocurrents, which implies the position of the laser beam. 91 This limits the post-processing methods of the PSD-based system, although it provides a very fast speed. Another drawback of PSD-based systems is that the high resolution of such systems relies on the assumption that the two analogue photocurrents should be amplified with two identical amplifiers, which is difficult to be achieved at a low cost. On the other hand, the CCD stores the intensity of the laser beam into an array of pixels. Many smart post-processing algorithms can then be applied to compute the laser beam position, including centroid, squared-centroid, fitting, Fourier, edge detection, etc. One of the issues with the CCD-based system is its low speed, caused not only by the longer hardware response time, but also the post-processing algorithm complexity. However, the flexibility of CCD-based systems provides greater freedom to balance performance (e.g., the image window size, laser beam intensity, and threshold, etc.).

This paper first compares the principles of both methods. Based on these principles, two key performance indicators, resolution and speed, are analyzed with particular attention paid to the issue of sensor saturation, which is a key fea111 ture that may result in unexpected errors, as illustrated at the 112 end of Sec. 3. During the discussion, various performance 113 improvements are proposed for both systems. According to 114 these improvements, experiments have been performed to as115 sess the performance with different parameters compared to 116 the state-of-the-art PSD-based system. Experimental results 117 and analysis are presented in Secs. 5 and 6.

\section{${ }_{118} 2$ Principle}

\section{2.1 PSD-Based Laser Beam Displacement $120 \quad$ Measurement}

121 The principle of a 1D PSD is shown in Fig. 1(a). The PSD has 122 a single active area formed by a P-N junction. The two parts 123 that originated from the laser spot to the two electrodes form 124 two lateral resistances for the photocurrents running toward 125 the electrodes. The photocurrents are collected through the 126 resistances by the output electrodes, which are inversely pro127 portional to the distance between the electrode and the center 128 of the incoming light beam. This relationship is expressed as

$$
x=\frac{L}{2} \frac{I_{2}-I_{1}}{I_{2}+I_{1}}
$$

130 where $I_{1}$ and $I_{2}$ are the electrode photocurrents, $L$ is the 131 length of the PSD active area and $x$ stands for the laser spot 132 position. According to the principle, the center given by a ${ }_{133}$ 1D PSD is the gravity center of incident light. The post134 processing process, which is typically implemented by the 135 external processing circuit, first amplifies each of the pho136 tocurrents, transfers them into voltages, and then calculates 137 the subtraction, addition, and division operations in Eq. (1).

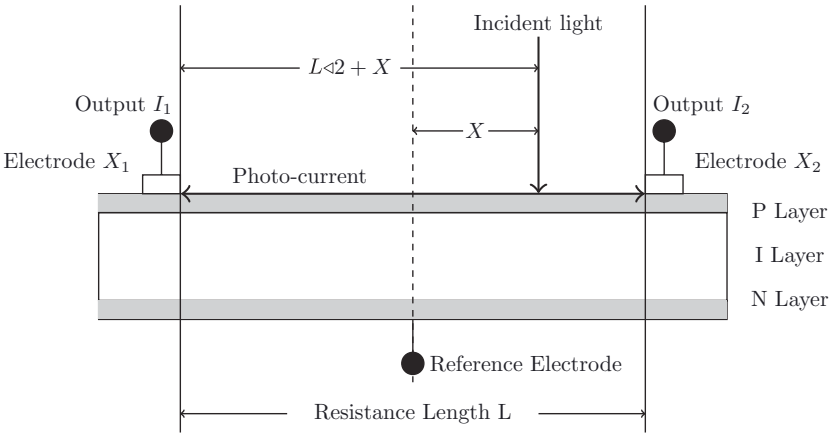

(a)

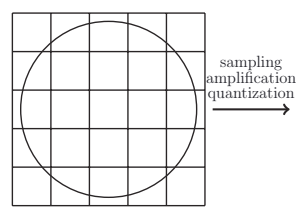

CCD sensor with Gaussian spot

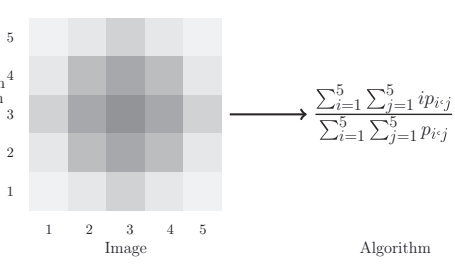

(b)
Fig. 1 The operating principles of both 1D PSD and CCD. (a) The operating principle of 1D PSD. (b) The operating principle of CCDbased system, a Gaussian beam (shown as the circle) shoot on a $5 \times 5$ pixels CCD. After sampling, amplification and quantization, the image records the power distribution of the Gaussian spot. An algorithm is then utilized to retrieve the position of the spot from the image.

\subsection{CCD-Based Laser Beam Displacement Measurement}

Figure 1(b) shows the operating principle of a CCD-based ${ }_{140}$ system. The image-capture process of a CCD-based sys- ${ }_{141}$ tem contains sampling, amplification, and quantization. It ${ }_{142}$ first samples the optical intensity and then converts the sam- ${ }_{143}$ ple into a signal charge, which is transformed into voltage ${ }_{144}$ through a common output. After voltage quantization, all sig- $\quad 145$ nals are processed by the post-processing algorithm to cal- 146 culate the position. As mentioned in Sec. 1, many algorithms $\quad 147$ can be used for laser beam position calculation. Algorithm 148 selection is based on the definition of the laser beam posi- ${ }_{149}$ tion, which highly depends on the applications. To compare 150 with a PSD, the definition of the laser beam position should ${ }_{151}$ be defined as the same as the PSD-based system, which is 152 the gravity center of the laser spot. Therefore, the centroid ${ }_{153}$ algorithm has been used here to calculate the gravity center ${ }_{154}$ due to its simplicity.

To analyze the resolution of the CCD-based system, a ${ }_{156}$ laser beam distribution model should first be introduced. A ${ }_{157}$ Gaussian beam is commonly used as a laser intensity model. ${ }_{158}$ A Gaussian beam $g(x, y)$ with peak intensity $I_{0}$ and beam ${ }_{159}$ waist $r_{0}$ is expressed as:

$g(x, y)=I_{0} e^{-x^{2}+y^{2} / 2 r_{0}^{2}}$.

After sampling by the pixels of the CCD, we can get the ${ }_{161}$ sampled signal:

$p(x, y)=g(x, y) s(x, y)=$

$$
\sum_{j_{1}=-\infty}^{\infty} \sum_{j_{2}=-\infty}^{\infty} g\left(j_{1} \Delta x, j_{2} \Delta y\right) \sigma\left(x-j_{1} \Delta x, y-j_{2} \Delta y\right)
$$

where $p(x, y)$ is the sampled signal, and $s(x, y)$ is the sam- 163 pling function. Here $\sigma$ stands for the dirac function. After 164 
165 sampling, the signal is quantized using different methods. To 166 simplify, we leave this point aside in this paper. According to 167 the centroid algorithm, the 1D center of the laser spot could 168 be expressed as:

$$
x=\frac{\sum_{i=-\infty}^{\infty} \sum_{j=-\infty}^{\infty} i p_{i, j}}{\sum_{i=-\infty}^{\infty} \sum_{j=-\infty}^{\infty} p_{i, j}} .
$$

169 From Eq. (3), the sums in Eq. (4) can then be replaced by 170 the integral, and the centroid of the function $p(x, y)$ can be 171 represented as follows:

$$
x=\frac{\int_{i=-\infty}^{\infty} \int_{j=-\infty}^{\infty} i p(x, y) d x d y}{\int_{i=-\infty}^{\infty} \int_{j=-\infty}^{\infty} p(x, y) d x d y},
$$

172 which results in the following formula by Fourier Q3 173 transformation: ${ }^{19}$

$$
x=-\frac{P_{u}^{\prime}(0,0)}{2 \pi j P(0,0)},
$$

174 where $P_{u}(x, y)$ is the Fourier transform of $p(x, y)$, and $175 P_{u}^{\prime}(x, y)$ is the derivative of $P_{u}$.

\section{Performance Analysis}

\subsection{Resolution}

178

$$
x_{d}=\bar{x}_{0}+d+e_{c} .
$$

${ }_{186}$ The systematic error $e_{c}$ caused by the sampling, quantization, 187 and centroid algorithm could be calculated by substituting 188 Eqs. (6) and (7) and used for correcting the resolution in 189 the post-processing phase. The resolution of the centroid 190 algorithm is also determined by the noise of the image sensor, 191 which includes three types of noises: the readout noise $N_{r}$, the 192 signal photon noise $N_{p}$, and the background noise $N_{b}$. The 193 background noise and the readout noise impinge all the pixels 194 in the image window; in contrast, the signal photon noise just 195 impacts the pixels illuminated by the laser spot. Since the 196 centroid algorithm processes all the pixels of the image, the 197 centers of both the laser spot and noises are calculated. If the 198 laser spot moves a distance $d$ from $x_{0}$, the calculated center $199 x_{d}^{\prime}$ is:

$$
x_{d}{ }^{\prime}=\frac{x_{r} M_{r n}+x_{b} M_{b n}+x_{p} M_{p n}+\left(x_{0}+d+e_{c}\right) M_{s}}{M_{r n}+M_{b n}+M_{p n}+M_{s}},
$$

200 where $x_{r}, x_{b}$, and $x_{p}$ are the centers of readout noise, back201 ground noise, and light photon noise, respectively, while $M_{r n}$, $202 M_{b n}, M_{p n}$, and $M_{s}$ respectively represent the mass of readout 203 noise, background noise, light photon noise, and laser spot. 204 As a reference, in a noisy environment, the equation that 205 expresses the original position $x_{0}{ }^{\prime}$ is listed below:

$$
x_{0}^{\prime}=\frac{x_{r_{0}} M_{r n}+x_{b_{0}} M_{b n}+x_{p_{0}} M_{p n}+x_{0} M_{s}}{M_{r n}+M_{b n}+M_{p n}+M_{s}},
$$

${ }_{207}^{206}$ in which $x_{r_{0}}, x_{b_{0}}$, and $x_{p_{0}}$ are the original centers of read208 out noise, background noise, and light photo noise. The background noise and the readout noise follow the Poisson 209 distribution which can be approximately considered as a 210 Gaussian distribution. Since these two noises impact all 211 the image pixels, the center of noises is independent of 212 the laser spot. That is to say, the following relationship 213 holds: $x_{r_{0}}=x_{r}$ and $x_{b_{0}}=x_{b}$. On the other hand, the signal 214 photon noise is related to the laser spot intensity, thus the 215 assumption could be made that the moved distance of light 216 photon noise approximately equals the moved distance of 217 the laser spot $d$ or $x_{p}=x_{p_{0}}+d$. By substituting Eqs. (8) 218 and (9), the systematic error $e$ is shown as:

$e=\frac{e_{c} M_{s}-d\left(M_{r n}+M_{b n}\right)}{M_{r n}+M_{b n}+M_{p n}+M_{s}}$.

The ratio between the calculated distance $d^{\prime}$ and the moved 220 distance of the laser spot could be expressed as follows:

$\frac{d^{\prime}}{d}=1+\frac{e}{d}=\frac{M_{p n}+M_{s}+\frac{e_{c}}{d} M_{s}}{M_{r n}+M_{b n}+M_{p n}+M_{s}}$.

The systematic error caused by noise can be eliminated or at ${ }_{222}$ least reduced in both the capture and post-processing phases. 223 Applying a threshold in the post-processing algorithm can 224 efficiently eliminate the readout noise and background 225 noise. Another post-processing way to reduce noise is to 226 average it using multiple images. If $N$ images are used for ${ }_{227}$ the calculation, the noise variance will be dropped to $2 / \sqrt{N} . \quad 228$ Temperature also has a very close relationship with the 229 noise. Typically, the CCD temperature should be reduced 230 as much as possible. In the laser deviation measurement 231 system, the temperature increase caused by the laser also 232 increases the noise. In the capture phase, the use of a pulsed 233 laser and synchronized exposure can solve this problem. 234 In Secs. 4 and 5, experiments applying these methods to 235 improve the resolution are described.

For a PSD-based system, the systematic error is mainly 237 due to the amplifier asymmetry for the two current signals $I_{1} \quad 238$ and $I_{2}$, ambient light, physical construction of the detector 239 head, and y-axis displacement. ${ }^{20}$ Noise is also a key factor 240 that may impact the resolution, including thermal noise cur- 241 rent, shot noise, and amplifier noise. To improve the PSD 242 resolution, we can increase interelectrode resistance, which 243 results in a reduction of both amplifier and shot noise. An- 244 other way to improve the resolution is to use symmetric and 245 low noise operational amplifiers, but it results in quite an ex- 246 pensive external processing circuit. The error caused by the 247 background light can be eliminated using pulse amplitude 248 modulation, shown in Ref. 6.

\subsection{Speed}

250

Speed is another important feature for the deviation posi- 251 tion measurement. One of the indices to judge PSD speed is 252 response time, defined as the time during which the output 253 signal rises from $10 \%$ to $90 \%$ of its peak value. The PSD 254 response time depends mainly on the physical features of 255 the PSD, for example its interelectrode resistance and termi- 256 nal capacitance. Reverse voltage and wavelength of incident 257 light also affect the response time. For most advanced PSDs, 258 the response time can reach $3 \mu \mathrm{s}$. In practice, the PSD out- 259 put signal will be sampled and digitalized for calculation. To 260 reduce the error caused by noise, the average of more than 261 one sample should be calculated. In general, the time cost 262 
263 for a PSD-based system mainly depends on the sum of the 264 digitalized time and the computing time.

265 Compared to a PSD, the CCD image sensor cannot output 266 a continuous signal: instead, it generates a series of frames, 267 which is a snapshot of the laser beam spot. The overall pro268 cessing time $t$ of a separate frame can be approximately 269 calculated as:

$$
t=t_{e}+t_{r}+t_{s}+t_{l}+t_{p}
$$

270 in which $t_{e}$ is the exposure time, $t_{r}$ is the readout time, $t_{s}$ 271 is the time used for storing the image, $t_{l}$ is the image load 272 time, and $t_{p}$ stands for the algorithm processing time. The 273 readout time includes the time in which all the rows shift 274 into serial register, the time in which the pixels move to

Q4 275 the AD converter under clock control, and the time spent 276 by $\mathrm{AD}$ conversion and digitization. Exposure time must be 277 long enough for the image sensor capture. The readout time 278 depends critically on the clock frequency, window size, and 279 window position. After the image is generated, the centroid 280 of the algorithm will take time to process the image. Image 281 size, algorithm used, and processor frequency are the main 282 factors impacting the processing time. For an image with $283 \quad M \times N$ pixels, the time complexity of the centroid algorithm 284 is $O(M N)$.

285 Several techniques are available to reduce the processing 286 time of image sensors. The CCD with dual serial registers and 287 two amplifiers can speed up the readout time. A laser with 288 larger intensity could shorten the exposure time. The CCD 289 with region of interest and binning features could output 290 fewer pixels, which can also improve the post-processing 291 speed with the cost of lowering the resolution. Implementing 292 the algorithm in hardware is another efficient way to improve 293 the speed of CCD-based systems.

\section{3.3 Saturation}

295 Usually, for optical sensors, saturation results in a large 296 error and should definitely be avoided. In practice, the PSD 297 output current follows a good linearity with respect to the 298 laser spot position. If the incident light power is too high 299 and therefore the saturation occurs, the output current is no 300 longer linear with the laser spot position. Hence, the centroid 301 information cannot be retrieved as expected. The parameter 302 "photocurrent saturation" is defined as the total output 303 current when the whole active area of a PSD is illuminated, 304 and is considered to express the saturation performance of 305 the PSD. This value depends on the interelectrode resistance 306 of the PSD and the reverse voltage. ${ }^{21}$ A direct way to avoid 307 saturation is to reduce incident light intensity. A PSD with 308 small interelectrode resistance and high reverse voltage can 309 prevent saturation from occurring.

310 The saturation of the CCD is defined as the maximum 311 amount of charges that the image sensor pixel can collect. 12 This amount of charge a pixel can hold in routine operation 3 is called its full well capacity. ${ }^{22}$ One effect of saturation is 314 that the linearity relationship between the number of col315 lected charges and the received light intensity will not stand 316 near the full well capacity, which causes the output signal to 317 generate unexpected distortion. One possible solution to this 318 problem is to adjust the camera gain control so that the full bit 9 depth of the ADC does not span the linear full well capacity 320 of the camera. This makes the image show saturation be321 fore real saturation occurs. ${ }^{23}$ Another influence is blooming. 2 When the image pixel full well capacity is reached, the more generated charges or the charges that cannot be transferred ${ }_{323}$ will pollute the adjacent image areas. A typical phenomenon 324 of blooming is the appearance of a white streak or an erro- 325 neous pixel signal value near the high intensity pixels. With 326 respect to measuring the displacement of the laser beams, 327 blooming leads to unexpected and nonrecoverable results, so 328 it should be avoided at all cost. If the camera gain is carefully ${ }_{329}$ adjusted to limit the ADC work in the linear full well capac- 330 ity, blooming will not occur when saturation is just observed. ${ }_{331}$ For the application discussed in this paper, the gravity center ${ }_{332}$ of a given laser spot is highly related to laser spot intensity, hence, saturation should be avoided in the measurement.

\section{Experiment Setup}

335

In Secs. 2 and 3, the factors that might impact the resolution and speed of a CCD-based system, have been described, including the laser beam power, the number of frames used 33 to calculate the position, the image window size, binning, and threshold. To assess the impact of these parameters on 340 system performance, different experiments have been carried ${ }_{341}$ out. Since the resolution and speed of PSD-based systems 342 highly depend on the equipment and device themselves, a 343 commercial PSD [Hamamatsu S3932 (Ref. 24)] and external 344 processing circuits [Hamamatsu C3683-01(Ref. 25)] were 345 used in the following experiments for comparing to CCD- 34 based systems.

A diode laser at a wavelength of $635 \mathrm{~nm}$ was mounted on 348 a motorized three-dimension micro-positioner with a mini- 349 mum step size of $0.1 \mu \mathrm{m}$, which moved along the $x$ axis of 350 the image under the control of a computer. The laser was 351 directly pointed at a PSD or a DALSA camera, ${ }^{26}$ which of- ${ }_{352}$ fers a $1280 \times 960$ resolution and a small $3.75 \times 3.75 \mu$ m pixel ${ }_{353}$ size. In order to control the power of the laser, a polarizer and 354 a filter were set up between the laser and the camera. This 355 setup is shown in Fig. 2. The CCD is removable and can be 356 replaced by a PSD.

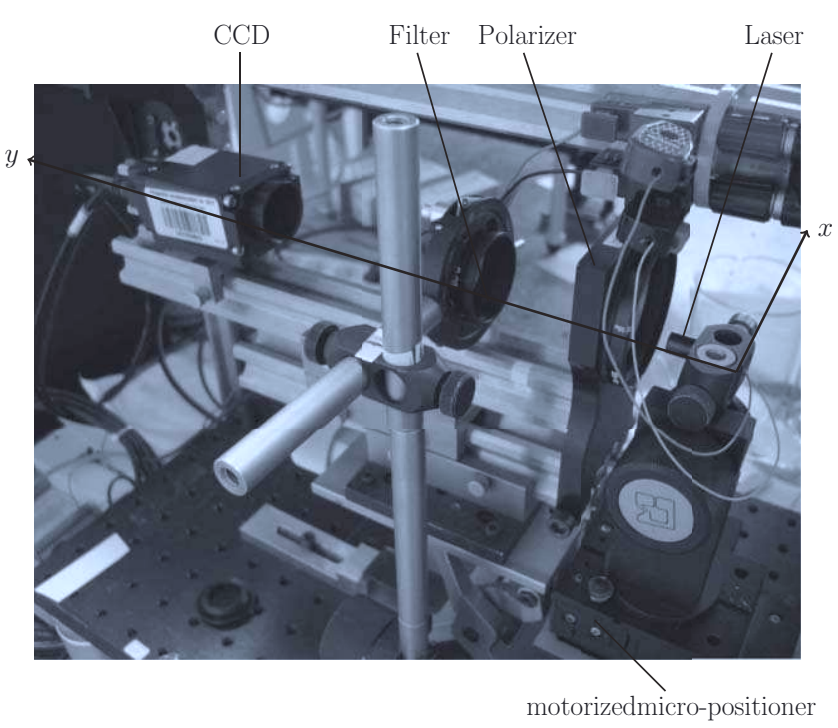

Fig. 2 The experiment setup. The laser is mounted on a motorized micro-positioner, which can move along the $x$ direction of the image. The laser is directly pointed at the CCD, which is removable and can be replaced by a PSD. 


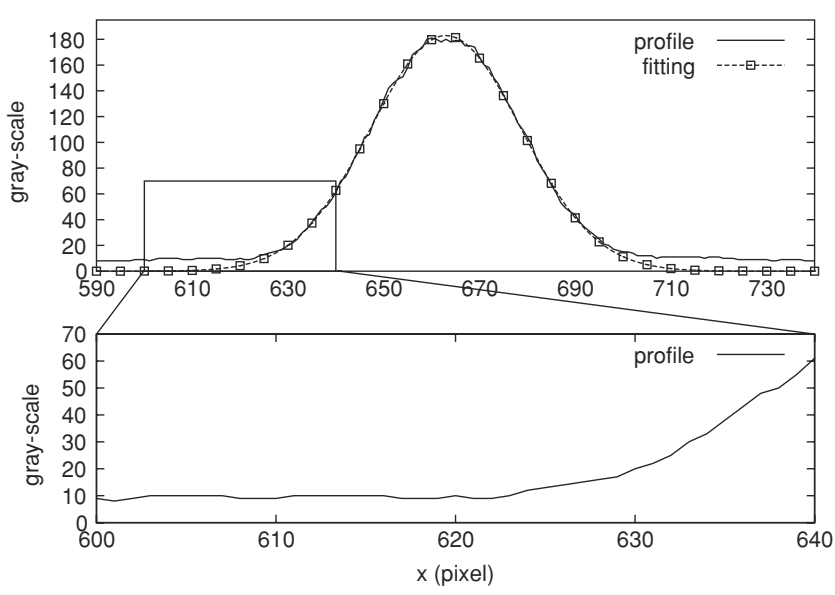

Fig. 3 The upper diagram plots the profile and Gaussian fitting of a laser beam; the lower diagram is the zoom view of the profile between 600 and 640 pixels.

Before comparing the CCD- and PSD-based systems, the factors mentioned in Secs. 2 and 3, should be first considered.

361 An experiment was carried out to analyze these factors. Dur362 ing the experiment, the laser was moved to a specific position 363 and 100 full size images were taken in $4 \mathrm{~s}$. This experiment 364 was repeated 50 times to avoid accidental error. According to 365 these 50 groups of images, different numbers of images were 366 selected in each group and processed with different image 367 window sizes and thresholds. The resolution of the system ${ }_{68}$ is assessed from two aspects: systematic error and its uncer9 tainty. For assessing systematic error and its uncertainty, the average of the calculated centers (the estimate of position ${ }^{27}$ ) and standard deviations (the standard uncertainty ${ }^{27}$ ) of all the groups were plotted. The power of the laser beam was adjusted to avoid saturation and blooming, as shown in Fig. 3. From the bottom chart in Fig. 3, the gray-scale of the sum of background noise and readout noise reaches about nine. Since the center of the ideal Gaussian spot equals the gravity center of the Gaussian spot, a Gaussian fitting was used to obtain the reference position and the laser beam waist. The average Gaussian center of the images calculated by a Gaussian fitting algorithm in the 50 experiments is 663.69 pixels and the average waist of the laser spot is 15.574 pixels. With different parameters, the estimate of the laser spot position and standard uncertainty were calculated. The systematic error of the CCD-based system was assessed by comparing the estimate of the calculated center with the reference center, whereas the uncertainty was evaluated by the standard deviation of calculated centers. A smaller distance between calculated center and reference center gives a smaller systematic error, and a smaller standard uncertainty is obtained 390 by a smaller standard deviation.

\subsection{Number of Processed Images}

392 With the 50 groups of images, different numbers of images 393 were selected in each group, and the center of each image was 394 calculated with no threshold and full image size. The estimate 395 of the calculated center and standard uncertainty are depicted 396 in Fig. 4. Both the estimate of center and standard uncertainty 397 in this chart show a trend to stabilize as more images were 398 used. This stability could be attributed to the fact that more

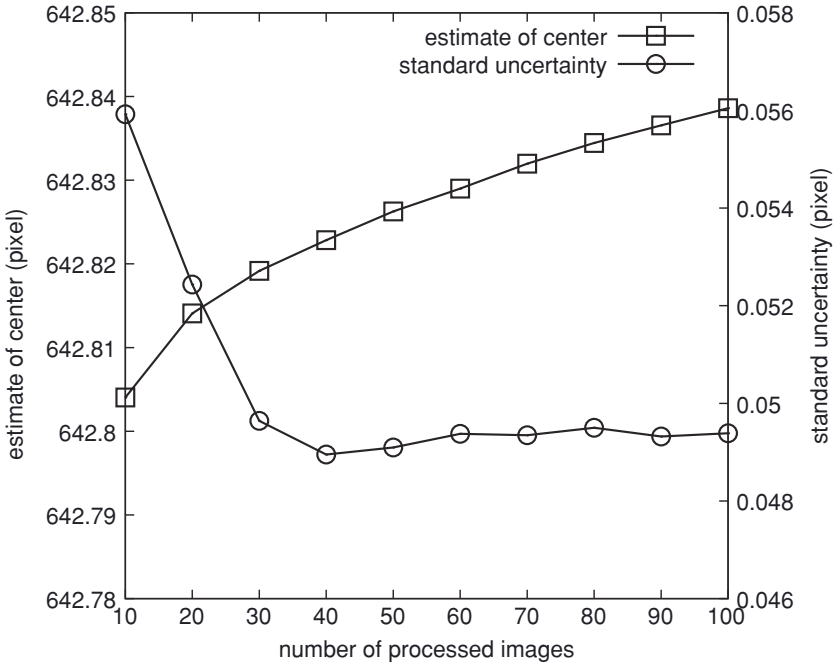

Fig. 4 The estimate of the center and the standard uncertainty of the systematic error against the number of processed images. The curve with squares is the estimate of the calculated center; the curve with circles is the standard uncertainty of the systematic error. The systematic error is assessed by comparing the estimate of the calculated center with the reference center (663.69 pixels). The original point of coordinate is the left bottom corner of the image.

images give more noise samples, making the center of noise 399 more stable. The standard uncertainty remains at a level of 400 less than 0.056 pixels, which gives a high level of precision. 401 However, the center given by the experiment is less than 402 642.9 pixels, which has a large deviation from the reference 403 center 663.69 pixels. According to Eq. (11), the large system- 404 atic error is caused by the noises that will lead to the measured 405 distance being much less than the actual distance. Although 406 the result shows a large systematic error, the variance of both 407 estimate of center and standard uncertainty caused by the 408 variance of the processed image number is quite small. That 409 is to say, the resolution of CCD-based systems do not highly 410 depend on the number of processed images. It is a good way ${ }_{411}$ to improve speed without greatly reducing resolution.

\subsection{Threshold}

As the number of processed images will not dramatically ${ }_{414}$ affect the resolution of a CCD-based system, 10 images 415 were selected for the calculation in the later experiment. ${ }_{416}$ As illustrated in Sec. 5.1, larger noise results in a larger ${ }_{417} \mathrm{Q} 7$ systematic error. Applying a threshold is a common method 418 to eliminate the effect of noises. With different thresholds 419 applied to the full-size images, the estimate of center and 420 standard uncertainty are plotted in Fig. 5. The behavior 421 of estimate and standard uncertainty is quite different ${ }_{422}$ between applying a threshold less than the level of noise and ${ }_{423}$ applying a threshold larger than the level of noises. When ${ }^{424}$ the threshold is less than the level of noise, the systematic ${ }_{425}$ error decreased but the standard uncertainty increased as the 426 threshold gets closer to the level of noise. Once the threshold ${ }_{427}$ is beyond the level of noise, both the estimate of center and ${ }_{428}$ standard uncertainty remain steady and the system reaches 429 high resolution (with estimate of calculated center $663.33 \quad 430$ pixels and standard uncertainty 0.045 pixels). 


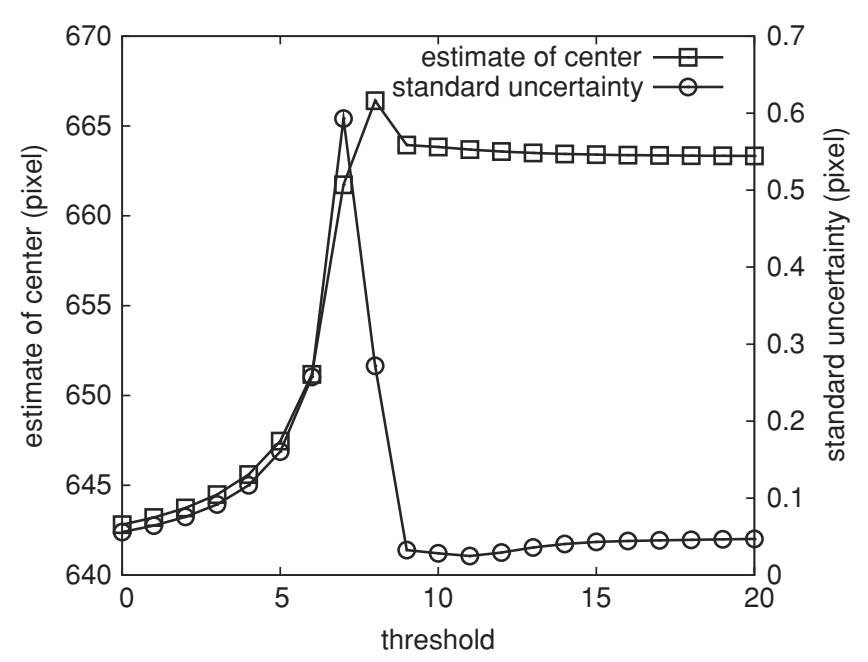

Fig. 5 The estimate of the center and the standard uncertainty of a systematic error against the threshold. The curve with squares is the estimate of the calculated center; the curve with circles indicates the standard uncertainty of a systematic error. The systematic error is assessed by comparing the estimate of the calculated center with the reference center (663.69 pixels). When the threshold is larger than the level of noise 9 , both the systematic error and the standard uncertainty are very small. The original point of coordinate is the left bottom corner of the image.

\section{${ }_{432}$ 5.3 Optimum Image Window Size}

433 Figure 6 describes how image window size affects resolution.

${ }_{434}$ The results are calculated with no threshold and 10 images.

435 It is obvious that both the systematic error and uncertainty 436 are sensitive to image window size. As the image window 437 size increased, a smaller uncertainty was obtained, while 438 the systematic error increased. One important reason that 439 might lead to the larger systematic error is the fact that the 440 mass of noises increased as the image window got larger. 441 The effect of the image window size mainly applies to the 442 standard uncertainty, which rises exponentially as the image

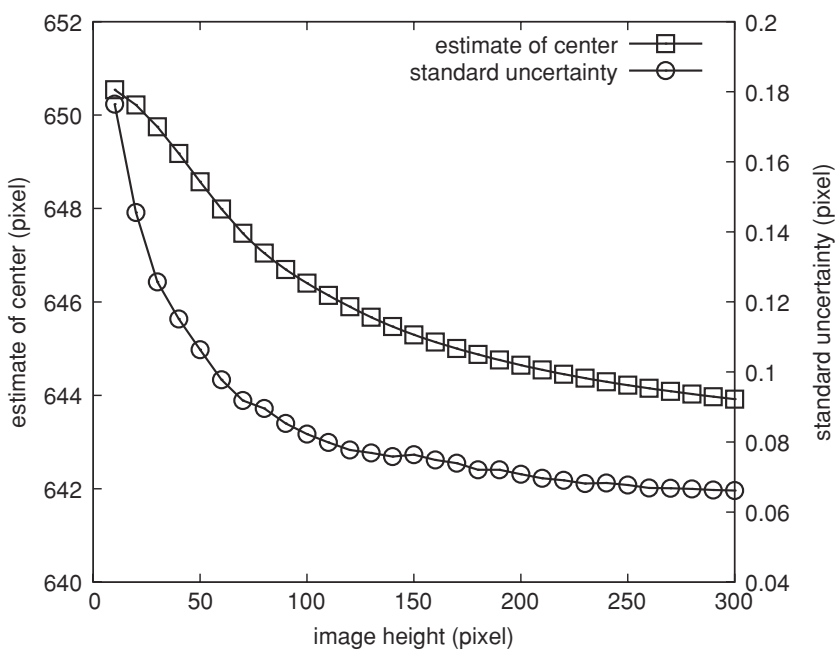

Fig. 6 The estimate of the center and the standard uncertainty of a systematic error against the height of image. The curve with a square is the estimate of the calculated center; the curve with a circle indicates the standard uncertainty of a systematic error. The systematic error is assessed by comparing the estimate of a calculated center with the reference center (663.69 pixels). The original point of the coordinate is the left bottom corner of the image.

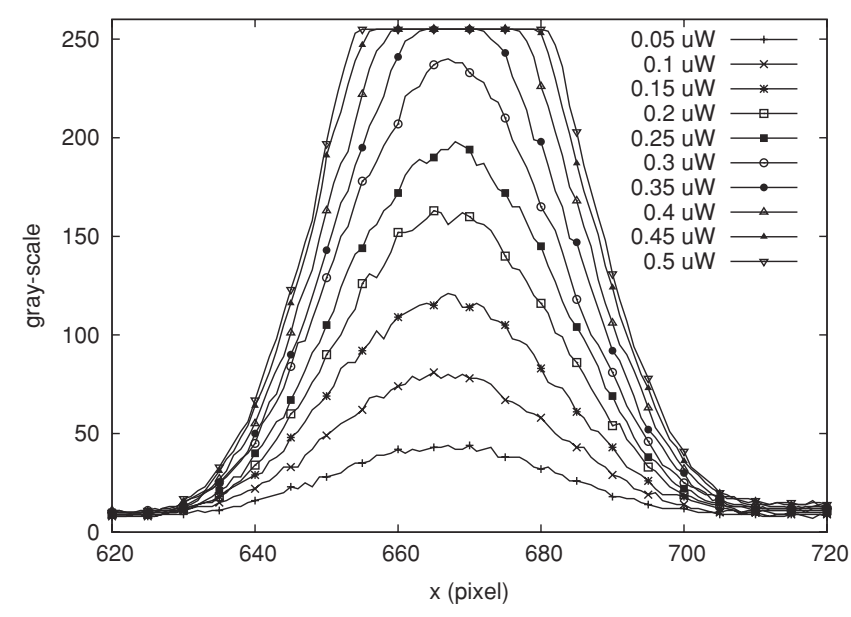

Fig. 7 Profiles of images with different laser beam powers.

window decreases linearly. To improve speed by using a ${ }_{443}$ smaller image window, the size of the image window should ${ }_{444}$ be carefully adjusted according to the performance required 445 from the application.

\subsection{Laser Beam Power and Saturation}

447

The power of the laser beam is related to the signal photon 448 noises, which overlay the Gaussian spot. An extremely high 449 laser beam power will lead to saturation or even blooming. $\quad 450$ All the signal photon noises, saturation, and blooming will 451 interfere with the systematic error and its uncertainty of a ${ }_{452}$ CCD-based system. To figure out the systematic error and 453 uncertainty according to different laser beam powers, an ex- ${ }_{454}$ periment was designed and implemented. Figure 7 shows the 455 profiles of images with different laser beam powers, and the ${ }_{456}$ estimate of centers and standard uncertainty are plotted in ${ }_{457}$ Fig. 8. The curve of the estimate of centers calculated with a ${ }_{458}$

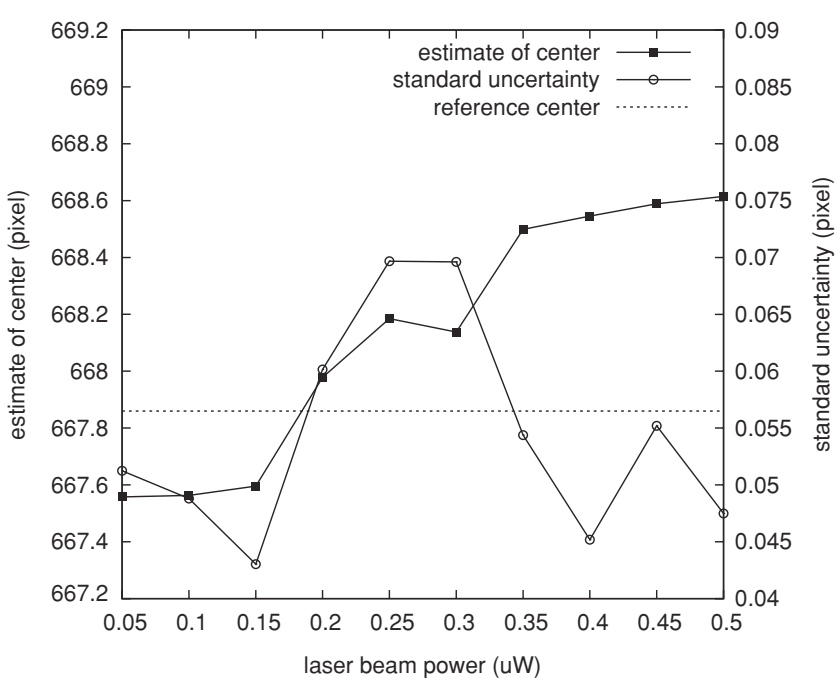

Fig. 8 The estimate of the center and the standard uncertainty of a systematic error against the power of the laser beam. The curve with squares is the estimate of the calculated center; the curve with circles is the standard uncertainty of a systematic error. The systematic error is assessed by comparing the estimate of a calculated center with the reference center. The dashed line indicates the reference center calculated by Gaussian fitting. The original point of coordinate is the left bottom corner of the image. 


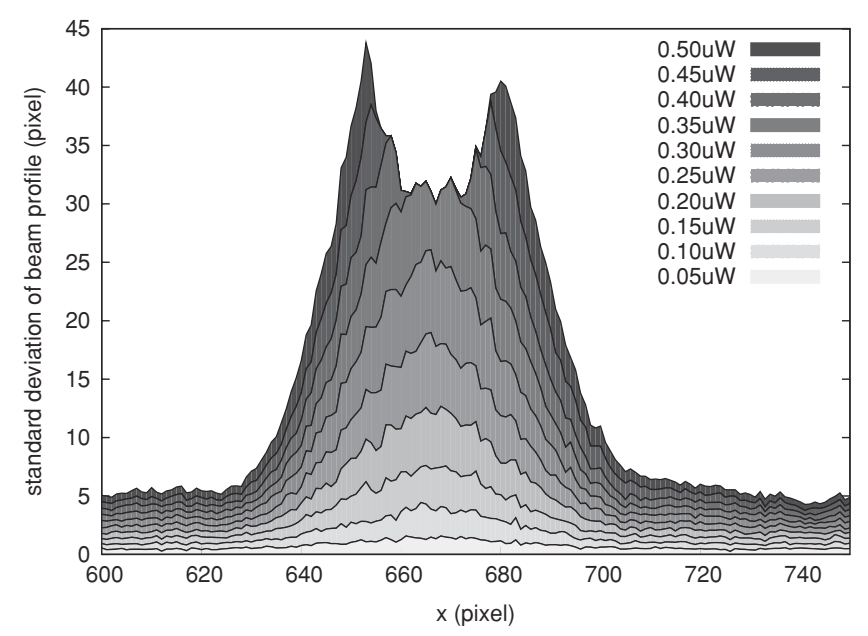

Fig. 9 The standard deviation of the laser beam profile in different powers, shown in different gray-scales.

460 two states. One state happens when the laser power is less

461 than $0.15 \mu \mathrm{W}$, while another occurs after a laser power larger

462 than $0.35 \mu \mathrm{W}$. Between these two states, the estimate of cen-

463 ter increases quickly as the laser power increases. The slight

${ }_{464}$ decrease of $0.3 \mu \mathrm{W}$ is possibly caused by accidental error.

465 The formation of the first state is due to the low noises brought

466 by the low laser power, which can be observed in Fig. 9. The

467 low standard uncertainty at the same phase further proves

468 this explanation. These noises affect the systematic error in

469 this state, which shows a difference between the reference

470 center (obtained by Gaussian fitting to the images with dif-

471 ferent powers, 667.86 pixels) and the calculated center. With

472 the increase in laser power, both the standard uncertainty

473 and the estimate of center increased quickly. This trend con-

474 tinues until saturation occurs at the power of $0.35 \mu \mathrm{W}$, as

475 shown in Fig. 7. From Fig. 9, it is clear that the noise rises

476 with the increase of laser power. This impacts the standard

477 uncertainty of the systematic error so that it gives a larger

478 uncertainty when the laser power increases. In addition to

479 noise impacting the systematic error, another factor that af-

480 fects the systematic error is the fact that the laser polarization

${ }_{31}$ might not be ideally uniform. This makes the Gaussian spot

${ }_{482}$ center move toward one direction when adjusting the power

483 by polarizer. Compared with Figs. 7 and 8 , it is obvious that

484 the second state is due to saturation, which hides most of the

485 signals and noises at the top of the laser spot intensity. Since

486 the noises are hidden by saturation, the standard uncertainty

${ }_{487}$ reduced to the level of the first state. Both low laser power

488 and saturation provide the system a small uncertainty and a

489 large systematic error. To obtain a smaller systematic error,

490 the power of the laser should be adjusted appropriately to

491 avoid a low signal-to-noise ratio and saturation.

\section{Comparison}

493 To compare the two different methods, the CCD image win494 dow size was cut to be the same size of the PSD, that is

$4954.8 \times 1 \mathrm{~mm}(1280 \times 267$ pixels $)$. The response of the two meth-

496 ods to various factors was taken by moving the motorized

497 micro-positioner with a step of $0.1 \mu \mathrm{m}$, which is considered

498 to be the reference measure to compare the two methods.

499 During each step, 10,000 samples of PSD signals and 64
Table 1 The slopes for different zones with CCD and PSD.

\begin{tabular}{lccc}
\hline \hline & $0 \mathrm{~mm}$ & $+0.5 \mathrm{~mm}$ & $+1 \mathrm{~mm}$ \\
\hline $\mathrm{CCD}(\mathrm{pixel} / \mu \mathrm{m})$ & 0.2622 & 0.2462 & 0.2625 \\
$\mathrm{PSD}(\mathrm{V} / \mu \mathrm{m})$ & 0.0019 & 0.0018 & 0.0017 \\
\hline \hline
\end{tabular}

images were captured in 1 second. For PSD, the laser beam 500 position was calculated by averaging the 10,000 PSD signal 501 samples, while the average image of 65 images was used to 502 compute the laser beam position by the centroid algorithm 503 with threshold 10 applied. To obtain a full-range compari- 504 son, five zones that are oriented from the center of the sensor 505 were chosen to perform the measurement. In each zone, the ${ }_{506}$ micro-positioner was moved by 50 steps. To guarantee that 507 a complete laser beam is contained in the active sensor sur- 508 face, the range of the measurement is set to the range of -1 to 509 $+1 \mathrm{~mm}$ according to the laser beam waist (46.6 pixels in this 510 experiment). The laser was synchronized with the camera 511 exposure by a National Instrument DAQ card in pulse mode. 512 The CCD saturation is avoided by applying a polarizer to re- 513 duce the laser power and setting the exposure time to $13 \mu \mathrm{s}$. 514 All the experiments are carried out in a dark room to obtain 515 the best performance.

\subsection{Resolution}

For comparing the resolution of both methods, these centers 518 for PSD, and CCD-based systems with different units were 519 converted to distance. The slope of the best fit line indicates 520 the ratio between the measurement unit and the distance. ${ }_{521}$ Thus the slopes are used to convert the measurement units 522 to distances which are listed in Table 1. In this table, the ${ }_{523}$ slopes for a CCD-based system are very close to the inverse ${ }_{524}$ of the image pixel size $(0.267 \mathrm{pixel} / \mu \mathrm{m})$, which shows that ${ }_{525}$ the systematic error of the CCD-based system is very small ${ }_{526}$ according to Eq. (11). The uncertainty of the systematic error ${ }_{527}$ can be evaluated by the standard deviation of the error ac- ${ }_{528}$ cording to the best fit line. The estimate of calculated centers ${ }_{529}$ and the error according to the best fit line of both a CCD and ${ }_{530}$ a PSD in each measurement zone are depicted by Fig. 10, 531 which shows good linearity for all the zones. The chart in the ${ }_{532}$ second row of Fig. 10 shows that the error of the PSD-based ${ }_{533}$ system is larger than that of the CCD-based system. The ${ }_{534}$ standard uncertainty of the errors in each zone are plotted 535 in Fig. 11, from which we can observe that the uncertainty 536 of the PSD-based system will gradually increase as the laser ${ }_{537}$ beam leaves the center. In contrast, the CCD-based system ${ }_{538}$ presents a good consistency in all the positions, with an av- 539 erage standard uncertainty $\pm 0.068 \mu \mathrm{m}$. It is obvious that the ${ }_{540}$ uncertainty of the CCD-based system is much less than the ${ }_{541}$ uncertainty of the PSD-based system (average standard un- 542 certainty $\pm 0.1077 \mu \mathrm{m})$ under the same operating conditions. ${ }_{543}$ And the resolution of the CCD-based system is insensitive to ${ }_{544}$ the laser spot position compared to the PSD-based system. $\quad 545$

\subsection{Speed}

546

During the experiment, the duration of both capture and pro- ${ }_{547}$ cessing was recorded simultaneously. The program was im- ${ }_{548}$ plemented in C and was carried out in a DELL notebook 549 LATITUDE E6500. For the PSD-based system, the duration ${ }_{550}$ 

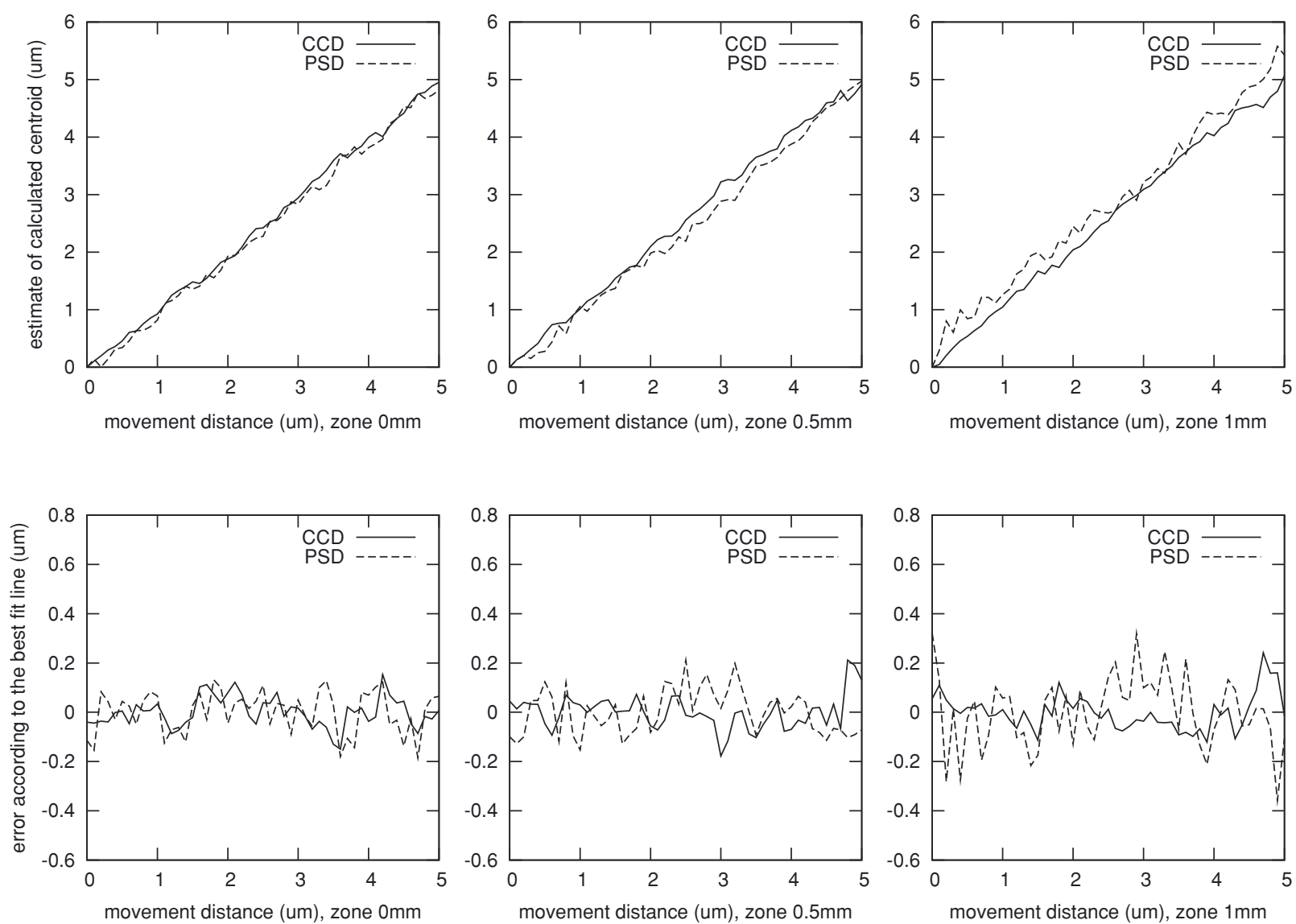

Fig. 10 The estimate of the center and the error according to the best fit line against the distance moved. The first row shows the estimate of the calculated centroid for a CCD and a PSD. The second row shows the error for a CCD and a PSD according to the best fit line. From left to right, the position of $0 \mathrm{~mm}$, the position of $+0.5 \mathrm{~mm}$, and the position of $+1 \mathrm{~mm}$.

551 contains not only the time used for capturing, sampling, and 552 digitalizing, but also the time elapsed for calculating the av553 erage. In addition to these, the image store and load time are 554 also considered for the CCD-based system. Figure 12 shows 555 the time cost to obtain one laser beam position for both of

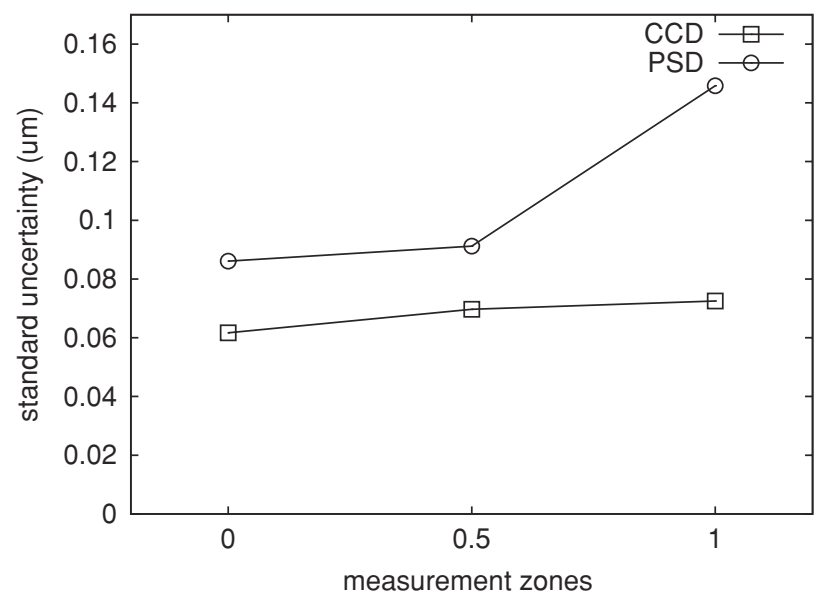

Fig. 11 The standard uncertainty of a systematic error. The first row shows the result with a CCD, the second row shows the result of a PSD. The systematic error is assessed by comparing the estimate of the calculated center with the best fit line. From left to right, the position of $0 \mathrm{~mm}$, the position of $+0.5 \mathrm{~mm}$, and the position of $+1 \mathrm{~mm}$ the systems. The time cost by PSD processing is very short, 556 with an average of $0.35 \mathrm{~ms}$. The primary time cost of the 557 PSD-based system is capture, which highly depends on the ${ }_{558}$ sample rate of the external circuit. Compared with the stabil- $\quad 559$ ity of the time cost associated with the PSD-based system, 560 the time cost of the CCD-based system depends greatly on ${ }_{561}$ the storage access time (store and load), which takes 80.4\% 562 of the total time on average due to the low speed of the 563
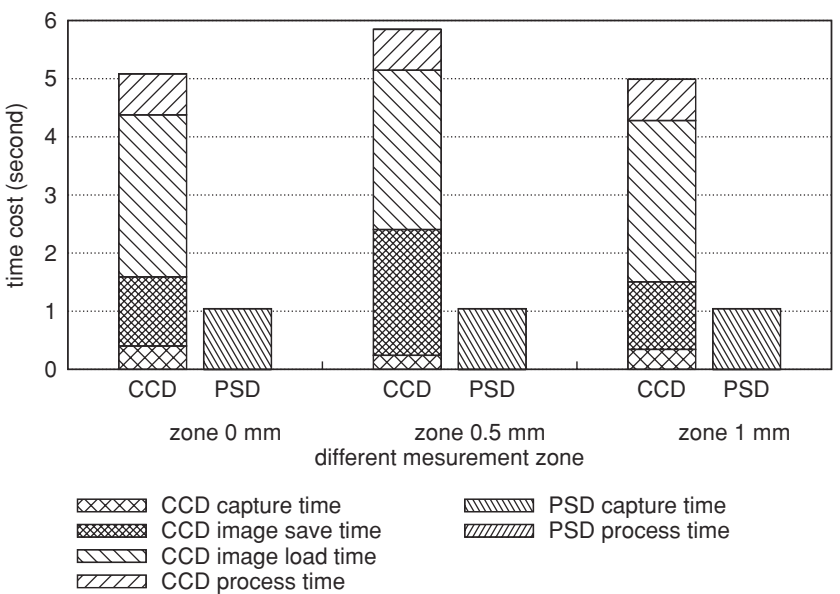

Fig. 12 Time cost in both a CCD-based system and a PSD-based system. 


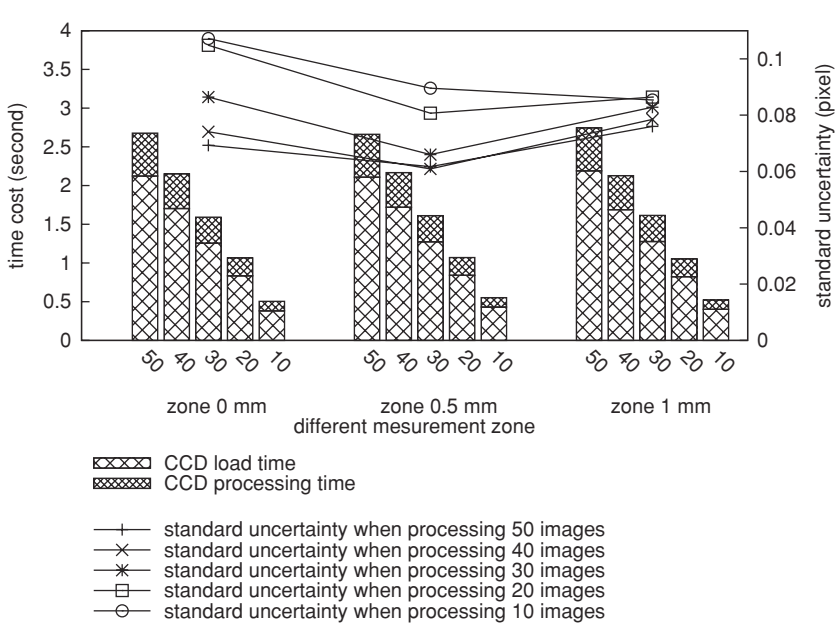

Fig. 13 Uncertainty and time cost for processing different numbers of images. The bars in the graph indicate the time cost with different numbers of processed images in different zones. The lines present the standard uncertainty of the systematic error with different numbers of processed images in different zones.

564 storage device. A possible improvement is to store all the 565 images in memory, which is much faster than the external 566 storage device. Furthermore, the processing time will also be 567 reduced due to the fast readout speed of memory. Although 568 the actual processing time reaches about $0.7 \mathrm{~s}$ on average, 569 which is much longer than the PSD-based system, it only 570 takes $13 \%$ of the total time. The time cost on capture and 571 processing is $1.036 \mathrm{~s}$, which nearly equals the time cost of a 52 PSD-based system (1.044 s). Thus, the critical time cost by 573 a CCD-based system is the storage access time, which needs 574 to be optimized in the system design and implementation.

575 6.3 Performance Trade-Off of CCD-Based System

\subsubsection{Number of images processed}

577 As discussed in Sec. 3.2, the time complexity of processing $578 \mathrm{~K}$ images with the size of $M \times N$ is $O(K M N)$. The time cost 579 by image processing is related to both the number of im580 ages and the image size. In previous experiments, 65 images 581 were used to calculate the center of a laser beam. With the 582 same image set, different numbers of images were selected 583 and used to calculate the center. The standard uncertainty 584 of systematic errors and time costs for this processing are 585 shown in Fig. 13. Time cost is a position-insensitive quantity, 586 which decreases linearly as the number of processed images 587 is reduced. With the decrease of processed images, the cal588 culated centers indicate a tendency toward growth. When 10 589 images were used for the centroid calculation, the time cost 590 by loading and processing images decreased to $0.53 \mathrm{~s}$ on aver591 age and the standard uncertainty of error rise to $\pm 0.094 \mu \mathrm{m}$, 592 which increased by $36.2 \%$ compared with the standard un593 certainty of error of processing 50 images (average standard 594 uncertainty of error is $0.069 \mu \mathrm{m}$ ). However, this uncertainty 595 is still smaller than the uncertainty of the PSD-based system

Q8 596 mentioned in Sec. block block. Although fewer images are 597 processed, the uncertainty of the systematic error will in598 crease, and the number of processed images will not greatly 599 reduce the resolution. In practical applications, reducing the 600 number of images is a good way to improve speed without 601 losing much resolution.

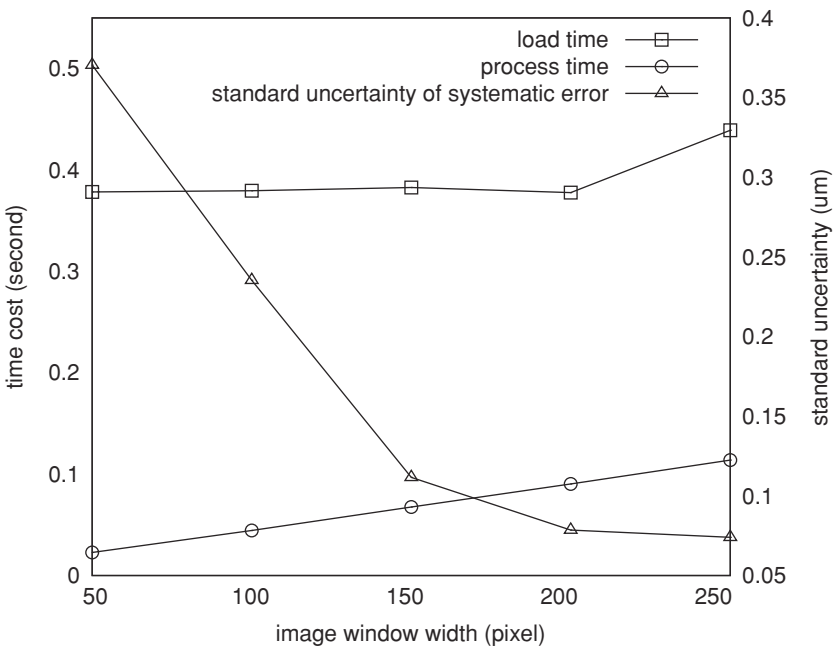

Fig. 14 Uncertainty and time cost for processing different image windows in position $0 \mathrm{~mm}$. The curve with squares is the time cost on loading the image for different image window widths. The curve with circles represents the process time for different image window widths. The curve with triangles indicates the standard uncertainty of the systematic error according to the image window width.

\subsubsection{Image window}

Another way to reduce the processing time is to diminish the 603 image size. To maintain the measurement range, the length 604 of the image window remained at $4.6 \mathrm{~mm}$ (1280 pixels) 605 and the width of the image window was diminished from 606 267 pixels to $250,200,150,100$, and 50 pixels. The same 607 image set was used but preprocessed by an image window 608 algorithm, which generates the desired image size. According 609 to the Sec. 6.3.1, 10 images were used for calculating the ${ }_{610} \mathrm{Q} 9$ position of the laser beam to obtain the worst resolution. The 611 uncertainty of the systematic error and time cost are depicted 612 in Fig. 14. As the image width decreased, the uncertainty 613 of the systematic error rose very quickly, from $0.074 \mu \mathrm{m}$ to 614 $0.37 \mu \mathrm{m}$. Compared with the speed improvement gained by 615 reducing the number of processed images, a smaller image ${ }_{616}$ window did not improve the speed remarkably. There is only 617 a benefit of $0.152 \mathrm{~s}$ in speed (load time and processing time), 618 with an increase of $0.3 \mu \mathrm{m}$ standard uncertainty. As the image 619 width decreases, the time cost decreases linearly, while the 620 uncertainty of the systematic error increases exponentially. 621 This shows a clear constraint for a CCD-based system: the 622 image window width should be larger than the size of the laser ${ }_{623}$ spot; otherwise, the resolution will decrease exponentially as 624 the image window width decreases.

\subsubsection{Binning}

626

Binning is the process of combining charges from adjacent ${ }_{627}$ pixels in a CCD during the readout phase. It will improve the ${ }_{628}$ readout speed at the expense of reducing the image dimen- ${ }_{629}$ sion in pixels (number of image pixels). Since the charge of 630 adjacent pixels will be read out at the same time, the read 631 out noise of the CCD working in binning mode will decrease 632 compared to the CCD working in non-binning mode. How- 633 ever, the background noise will increase due to a larger expo- 634 sure area per pixel unit. An experiment of binning was carried 635 out under the same environment as the previous experiments. $\quad 636$ A $2 \times 2$ binning was applied for the measurement, thus image 637 dimension reduced to $640 \times 133$ pixels. To verify the effect 638 


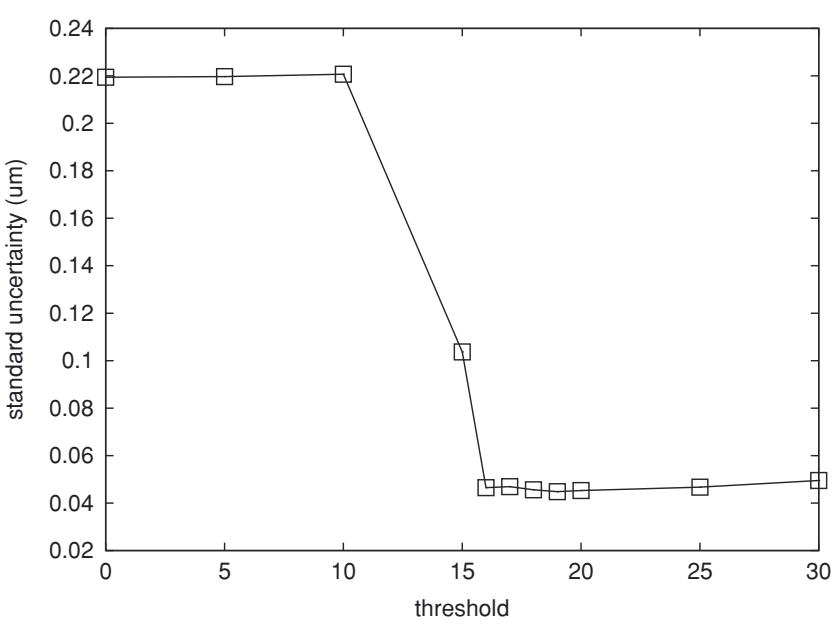

Fig. 15 Standard uncertainty of the systematic error for different thresholds. Binning will cause the level of noise increase when the background illumination is high. Applying a higher threshold can improve the resolution when using the binning technique.

639 of the noise, different thresholds were applied to 10 images 640 for each position. The standard uncertainty of the systematic 641 error are plotted in Fig. 15. The threshold 10, which was 642 applied for the previous experiments, is not effective enough 643 in the case of $2 \times 2$ binning. The standard uncertainty of the 644 systematic error $( \pm 0.2423 \mu \mathrm{m})$ for applying threshold 10 645 is nearly the same as the one without applying the threshold $646( \pm 0.2469 \mu \mathrm{m})$. However, with a threshold of 19 , the standard 647 uncertainty reduced to the minimum value $\pm 0.0448 \mu \mathrm{m}$, af 648 ter which the standard uncertainty of the systematic error 649 increased slightly as the threshold increased. Until applying 650 the threshold of 85 , the standard uncertainty remained at a 651 level of less than $0.1 \mu \mathrm{m}$. The average load time and process 652 time reduced to 0.3 and $0.1 \mathrm{~s}$ separately. Binning will lead 653 to a fast system speed with more noise when the background 654 illumination is high. Under a high illumination situation, the 655 binning technique should be used with a higher threshold to 656 maintain high resolution.

\section{Conclusion}

658 Both the PSD and the CCD provide the capability of measur659 ing laser beam deviations. As an analogue device, the PSD 660 simply outputs two photocurrents to represent incident light 661 position. The CCD utilizes a series of pixels which form a 662 2D image window to record the incident light intensity dis663 tribution. This 2D image window provides more information 664 for post-processing. A narrow image window loses a lot of 665 information, while a wide image window brings more noise 666 and computation load. The optimal image window can be 667 decided by the performance requirement of the application. 668 In this paper, measurement achieved in a clear medium in 669 a lab environment shows that the average resolution of a 670 CCD-based system is nearly 1.5 times the average resolu671 tion of a PSD-based system. Furthermore, the resolution of a 672 CCD-based system is independent of the incident laser beam 673 position, while the PSD-based system obtains worse resolu674 tion as the laser beam position gets farther from the center of 675 the PSD.

676 The performance of a CCD-based system could be 677 adjusted by adjusting different parameters. A small image window size is useful not only for increasing the resolution 678 but also for improving the speed with the limitation that 679 the laser spot should be entirely contained in the image. 680 Applying a threshold to the noise level could efficiently 681 reduce the systematic error. To improve the speed, binning 682 is an alternative means, but the noise level should be 683 reconsidered to choose a proper threshold. Saturation hides a 684 lot of the laser spot information and thus should be definitely 685 avoided. According to the analysis and experiment results 686 provided in this paper, a CCD-based system can obtain better ${ }_{687}$ resolution than a PSD-based system with a comparable 688 speed to a PSD-based system by adjusting these parameters. 689 This makes the CCD a better alternative to the PSD in beam 690 deviation measurement applications. Furthermore, the CCD 691 records all the power distribution information of the laser 692 spot, thus giving the capability of measuring the power 693 distribution sensitive quantities, such as the turbidity of 694 seawater.

1. D. Malardé, Z. Y. Wu, P. Grosso, J.-L. de Bougrenet de la Tocnaye, and M. Le Menn, "High-resolution and compact refractormeter for salinity measurements," Meas. Sc. Technol. 1, 20 (2009).

2. H. Canabal, J. Alonso, and E. Bernabeu, "Laser beam deflectometry based on a subpixel resolution algorithm," Opt. Eng. 40, 2517 (2001).

3. J. T. Wallmark, "A new semiconductor photocell using lateral photoeffect," Proceedings of the Institute of Radio Engineers, Vol. 45, pp. 474-483 (1957)

4. H. X. Song, X. D. Wang, L. Q. Ma, M. Z. Cai, and T. Z. Cao, "Design and performance analysis of laser displacement sensor based on position sensitive detector (PSD)," J. Phys. 48(1), 217-222 (2006).

5. Z. Huili, H. Xiaorui, Z. Yuning, and W. Xuanze, "Research on position detection of psd based on light intensity modulation and digital fit," Int. J. Intell. Syst. 3, 38-42 (2009).

6. W. Guanghui, P. Shum, X. Guoliang, Z. Xuping, "Position detection improvement of position sensitive detector (psd) by using analog and digital signal processing," 2007 6th International Conference on Information, Communications \& Signal Processing, pp. 1-4 (2007). 7. G. Philippe, M. Damien, M. Le Menn, W. Zongyan, and J.-L.
de Bougrenet de La Tocnaye, "Refractometer resolution limits for measuring seawater refractive index," Opt. Eng. 49(10) (2010).

8. P. F. I. Scott, A. S. Kachatkou, N. R. Kyele, and R. G. van Silfhout, "Real-time photon beam localization methods using high-resolution imagers and parallel processing using a reconfigurable system," Opt. Eng. 48, (2009).

9. S. S. Welch, "Effects of window size and shape on accuracy of subpixel centroid estimation of target images," NASA Technical Paper 3331, September 1993.

10. N. Bobroff, "Position measurement with a resolution and noiselimited instrument," Rev. Sci. Instrum. 57, 1152-1157 (1986).

11. M. R. Shortis, T. A. Clarke, and T. Short, "A comparison of some techniques for the subpixel location of discrete target images," Proc. SPIE 2350, 239-250 (1994).

12. T. A. Clarke and X. Wang, "Analysis of subpixel target location accuracy using Fourier-transform-based models," Proc. SPIE 2598, 7788 (1995).

13. R. Singh, J. M. Hattuniemi, and A. J. Mäkynen, "Analysis of accuracy of the laser spot centroid estimation," Proc. SPIE 7022 (2007).

14. B. F. Alexander and K. C. Ng, "Elimination of the systematic error in subpixel accuracy," Opt. Eng. 30, 1320-1331 (1991).

15. H. Chen and C. Rao, "Accuracy analysis on centroid estimation algorithm limited by photo noise for point object," Opt. Comm. 282(8), 1526-1530 (2009).

16. T. A. Clarke, M. A. R. Cooper, and J. G. Fryer, "An estimator for the random error in subpixel target location and its use in the bundle adjustment," Proc. SPIE 2252, 161-168 (1994).

17. J. W. Cui, J. B. Tan, L. Ao, and W. J. Kang, "Optimized algorithm of the laser spot center location in strong noise," J. Phys.: Conf. Ser. 13, 312-315 (2005).

18. I. Edwards, "Using photodetectors for position sensing," Sensors Magazine, Dec. 1988.

19. Bracewell and R. Newbold, The Fourier Transform and its Applications, McGraw-Hill, New York (1978).

20. S. Iqbal, M. M. S. Gualini, and A. Asundi, "Measurement accuracy of lateral-effect position-sensitive devices in presence of stray illumination noise," Sens. Actuators, A 143, 286-292 (2008).

21. HAMAMATSU PHOTONICS K.K., Solid State Division, Characteristics and use of psd. http://sales.hamamatsu.com/assets/pdf/ catsandguides/psd_technical_information.pdf. 
755 22. S. B. Howell, Handbook of CCD Astronomy, Cambridge University Q16 756

757 23. HAMAMATSU PHOTONICS K.K., Solid State Division. Ccd satu758 ration and blooming. http://learn.hamamatsu.com/articles/ ccdsatand759 blooming.html.

760 24. HAMAMATSU PHOTONICS K.K., Solid State Division. Onedimensional psd s3931, s3932, s3270. http://sales.hamamatsu.com/ assets/pdf/parts_S/s3931_etc_kspd1002e05.pdf.

25. HAMAMATSU PHOTONICS K.K., Solid State Division. Signal processing circuit for 1D psd c3683-01. http://sales.hamamatsu.com/ assets/pdf/parts_C/C3683-01.pdf.

766 26. DALSA Corporation. Genie m1280 datasheet, 2009. http://www. dalsa.com/prot/mv/datasheets/genie_m1280_1.3.pdf.
27. BIPM, IEC, IFCC, ISO, IUPAC, IUPAP, and OIML, "Guide to the 767 expression of uncertainty in measurement," ISO, Geneva, 1993.

Bo Hou is a PhD student at Telecom Bretagne, France. He received 769 his MS degree in computer science from Beijing University of Posts 770 and Telecommunications. He has worked for IBM China Develop- 771 ment Laboratory, researching and developing Business Intelligence 772 systems. His research interests include information systems, image 773 processing, and high-precision instrumentation.

Biographies and photographs of the other authors not available.

775 


\section{Queries}

Q1: Au: Please supply authors first names.

Q2: Au: Please check the placements of Ref. 18.

Q3: Au: Please check placement of Ref. 19.

Q4: Au: Please define AD.

Q5: Au: Please check change from "the previous section" to "Secs. 2 and 3".

Q6: Au: Please check change from "the previous section" to "Secs. 2 and 3".

Q7: Au: Please check change from "the previous section" to "Sec. 5.1".

Q8: Au: Please indicate to which section you are refering to in "last section".

Q9: Au: Please check change from "last section" to "Sec. 6.3.1".

Q10: Au: Please supply page range for Refs. 1, 2, 8.

Q11: Au: Please provide publisher/sponsor and location for Refs. 3 and 6

Q12: Au: Please check author names in Ref. 7 and supply page range.

Q13: Au: Please check Ref. 7 for content errors, or provide the DOI.

Q14: Au: Please supply volume or page range for Ref. 13

Q15: Au: Please supply initial of 1st author in Ref. 19.

Q16: Au: Please supply location of publisher in Ref. 22. 